\section{Barbara Kauffmann}

\section{Völkerrecht/ Vereinte Nationen}

Fröhlich, Manuel (Hrsg.): UN Studies. Umrisse eines Lehrund Forschungsfeldes. BadenBaden (Nomos Verlagsgesellschaft) 2008.

Ziolkowski, Katharina: Gerechtigkeitspostulate als Rechtfertigung von Kriegen. Zum Einfluss moderner Konzepte des Gerechten Krieges auf die völkerrechtliche Zulässigkeit zwischenstaatlicher Gewaltanwendung nach 1945. Baden-Baden (Nomos Verlagsgesellschaft) 2008 .

\section{Abrüstung/ Rüstungskon- trolle/ Militär/ Verteidi- gung}

Bredow, Wilfried von: Militär und Demokratie in Deutschland. Eine Einführung. Wiesbaden (Verlag für Sozialwissenschaften) 2008.

Hans J. Gießmann/Götz Neuneck (Hrsg.): Streitkräfte zähmen, Sicherheit schaffen, Frieden gewinnen. Festschrift für Reinhard Mutz. Baden-Baden (Nomos Verlagsgesellschaft) 2008.

Institute for Defense and Disarmament Studies (Hrsg.): A Chronicle of Treaties, Negotiations, Proposals, Weapons, and Policy. New York (Institute for Defense and Disarmament Studies) 2008.

Springer, Natalia: Die Deaktivierung des Krieges. Zur Demobilisierung von Gesellschaften nach Bürgerkriegen. Baden-Baden (Nomos Verlagsgesellschaft) 2008 .

\section{Nationalismus/ethnische Konflikte}

Visser, Reidar/Stansfield, Gareth (Hrsg.): An Iraq of its Regions. Cornerstones of a Federal Democracy? New York (Columbia University Press) 2008.

\section{Europa/ EU/ Osterweite- rung}

Rasch, Maximilian B.: The European Union at the United Nations. The Functioning and Coherence of EU External Representation in a StateCentric Environment. Leiden (Brill) 2008 .

Joerges, Christian/ Mahlmann, Matthias/ Preuß, Ulrich K. (Hrsg.): „Schmerzliche Erfahrungen aus der Vergangenheit" und der Prozess der Konstitutionalisierung Europas. Wiesbaden (Verlag für Sozialwissenschaften) 2008 .

Kühnhardt, Ludger: European Union. The Second Founding. The Changing Rationale of
European Integration. BadenBaden (Nomos Verlagsgesellschaft) 2008

McGowan, Lee/ Phinnemore, David: A Dictionary of the European Union. New York (Routledge) 2008.

Myant, Martin/ Cox,Terry (Hrsg.): Reinventing Poland. Economic and Political Transformation and Evolving National Identity. New York (Routledge) 2008.

Selck, Torsten/Veen, Tim(Hrsg.): Die politische Ökonomie des EU-Entscheidungsprozesses. Modelle und Anwendungen. Wiesbaden (Verlag für Sozialwissenschaften) 2008 .

Walter, Jochen: Die Türkei -, Das Ding auf der Schwelle'. (De-)Konstruktionen der Grenzen Europas. Wiesbaden (Verlag für Sozialwissenschaften) 2008.

Petritsch, Wolfgang / Solioz, Christophe (Eds), Regional Cooperation in South East Europe and Beyond, BadenBaden (Nomos) 2008.

\section{Außen- und Sicherheits- politik allgemein}

Freudenberg, Dirk: Theorie des Irregulären. Partisanen, Guerrilas und Terroristen im modernen Kleinkrieg. Wiesbaden (Verlag für Sozialwissenschaften) 2008.
Gärtner, Heinz (Hrsg.): Internationale Sicherheit. Definitionen von A - Z. Baden-Baden (Nomos Verlagsgesellschaft) 2008 .

Misra, Amalendu: Politics of Civil Wars. Conflict, Intervention and Resolution. New York (Routledge) 2008.

Heinemann-Grüder, Andreas /Jochen Hippler/Markus Weingardt/Reinhard Mutz/Bruno Schoch (Hrsg.) Friedensgutachten 2008. Münster (LIT) 2008.

\section{Sonstiges}

Hufbauer, Gary Clyde (Hrsg.): Economic Sanctions Reconsidered. Washington, DC (The Peterson Institute for International Economics) 2007.

Kovacevic, Natasa: Narrating Post/Communism. Colonial Discourse and Europe's Borderline Civilization. New York (Routledge) 2008.

Rear, Michael: Intervention, Ethnic Conflict and StateBuilding in Iraq. A Paradigm for the Post-Colonial State. New York (Routledge) 2008.

Warburg, Jens: Das Militär und seine Subjekte. Zur Soziologie des Krieges. Bielefeld (Transkript Verlag) 2008. 